Central European Journal of Energetic Materials, 2016, 13(2), 357-379

ISSN 1733-7178

e-ISSN 2353-1843

\title{
Desensitization by Pre-shocking in Heterogeneous Explosives and its Numerical Modelling
}

\author{
Tariq HUSSAIN*, Liu YAN**, Huang FENGLEI, Luo YI \\ State Key Laboratory of Explosion Science and Technology, \\ Beijing Institute of Technology, Beijing 100081, P. R. China \\ E-mails: "tariq_msc@yahoo.com, **liuyan@bit.edu.cn
}

\begin{abstract}
Desensitization caused by pre-shocking in heterogeneous explosives is discussed. The aim of this study was to find a simple numerical model that could reproduce the important features of previously reported shock desensitization experiments. After reviewing the previous experimental results and modelling efforts, an extension of the Lee-Tarver reactive flow model is proposed. The proposed desensitization model is based upon the experimentally determined desensitization criteria for explosives. The additional parameters required for this extension can be calibrated by experiment for a typical explosive. The new model has been implemented in the hydrodynamic code LS-DYNA as a user defined equation of state, and is now available to simulate various kinds of situations involving explosives up to the limits and capabilities of LS-DYNA. Desensitization by pre-shocking in double shock experiments, reflected shock and detonation quenching experiments have been studied using the new model, and the results were found to be in qualitative agreement with the experimental results reported in the literature.
\end{abstract}

Keywords: shock desensitization, desensitization criterion, ignition and growth, Lee-Tarver model, detonation quenching, reflected shock ignition, EDC37

\section{Introduction}

The term "shock desensitization" is usually referred to a variety of phenomena occurring in explosives such as dead pressing, detonation quenching and the reduced sensitivity of explosives to multiple shocks. It is now an established fact that passage of a weak shock desensitizes the explosive to a succeeding stronger shock. Although its chemical composition is almost unchanged by the first shock, 
the pre-compressed explosive requires a stronger shock for initiation compared to that required for initiation of the pristine, unshocked material. Usually a strong second shock does not cause reaction until it overtakes the first shock.

\section{Experimental Evidence}

The phenomenon of desensitization of heterogeneous explosives by pre-shocking has long been under investigation by many researchers. As early as 1960, Campbell et al. [1] reported that a $3.9 \mathrm{GPa}$ preshock desensitized a plasticbonded HMX explosive to initiation by a following $10 \mathrm{GPa}$ shock. In a later study, Campbell and Travis [2] desensitized PBX-9404 and Composition B-3 by subjecting them to pre-shocks in the pressure range 1.0-2.4 GPa. It was observed that desensitization occurred in a very brief time window. Using their experimental data, a relation was proposed between the pre-shock pressure $(P)$ and the time required $(\tau)$ for desensitization of PBX-9404 as:

$$
P^{2.2} \tau=1150\left(\mathrm{kbar}^{2.2} \cdot \mu \mathrm{s}\right)
$$

Another study [3] of the initiation and build-up to detonation of Composition B covered by steel plates of different thicknesses and impacted by a shaped-charge jet, is also evidence for desensitization by pre-shocking. Using laser velocity interferometry, Setchell [4] investigated the effects of precursor shocks (3.2 GPa sustained shock of duration $3.7 \mu$ s or a $2.0 \mathrm{GPa}$ ramp wave having a $0.6 \mu$ s rise time) propagating ahead of stronger $5.0 \mathrm{GPa}$ shock waves in a granular low porosity PBX-9404 explosive. The extension of the run distance to detonation depended on the duration of the precursor shock wave in both cases.

Mulford et al. [5] studied the effect of a sustained shock followed $0.65 \mu \mathrm{s}$ later by a second shock, on the initiation of PBX-9404. The run distance to detonation was extended and the initiation of detonation occurred nearly as anticipated by Pop-plots for PBX-9404, after the coalescence into a single wave of a $2.5 \mathrm{GPa}$ pre-shock and a $5.6 \mathrm{GPa}$ main shock. Slight growth of reaction was observed in the pre-shocked region on the passage of the second shock, indicating that the desensitization in the pre-shocked region was not perfect. This was in accordance with Equation 1, as in this case:

$$
P^{2.2} \tau=644\left(\mathrm{kbar}^{2.2} \cdot \mu \mathrm{s}\right)
$$

which is less than the experimentally determined [2] value for PBX-9404. 
Mulford and Swift [6] later developed a reactive flow model to reproduce the experimentally observed shock desensitization phenomenon.

Tarver et al. [7] observed the desensitization of LX-17 in reflected shock and colliding shock experiments, and numerically modelled this using the ignition and growth reactive flow model [8]. These pressure histories, measured by manganin pressure gauges, showed that LX-17 can be dead pressed at slightly lower pressures than the threshold (7 GPa).

Bordzilovskii and Karakhanov [9] experimentally studied multiple shock initiation of pressed RDX/paraffin and HMX/paraffin compounds and observed weak reaction behind the second shock wave. It was found that the run distance to detonation in the pre-shocked explosive was simply the sum of the distance at which the two shocks coalesced and the run to detonation distance expected for the second shock wave from Pop-plot data. The experimental work by Bat'kov et al. [10] also reveals the decrease in shock-wave sensitivity of pressed TNT and TNT/RDX compositions (50/50 and 30/70) caused by pre-shocking.

More recently, Vandersall et al. [11] performed shock desensitization and detonation quenching experiments on LX-17 using a Teflon ${ }^{\mathrm{TM}}$ flyer fired from a gas gun to impact one end of a cylindrical sample of LX-17 and to generate a single low amplitude shock wave to pre-shock the explosive sample just prior to detonating it from the other end. At pre-shock pressures of $\sim 0.68$ and $\sim 1.1 \mathrm{GPa}$, LX-17 detonation waves failed in the pre-shocked region, a process referred to as detonation quenching. However, at a pre-shock pressure of $\sim 0.52 \mathrm{GPa}$, the detonation wave proceeded through the sample, and no detonation quenching was observed. It was concluded that the lower limit for the desensitization shock strength lies between 0.52 to $0.68 \mathrm{GPa}$. However more experiments are needed to determine exactly the limits of the pressure range and the time window for desensitization.

A series of double shock initiation experiments $[12,13]$ on an HMX-based explosive EDC37 (91\% HMX and 9\% binder) revealed more interesting facts. In the first set of experiments (experimental shots 1175/1176), the explosive was subjected to a $2.87 \mathrm{GPa}$ shock followed by a $6.2 \mathrm{GPa}$ shock. No reaction was observed in the first wave, and the run to detonation distance after the waves coalesced was as expected from the single shock Pop-plot. However in the second set of experiments (shots 1194/1195), where the explosive was subjected to a $3.92 \mathrm{GPa}$ shock followed by a main shock of $8.56 \mathrm{GPa}$ strength, significant reaction occurred in the first wave and even more in the second wave. The run distance to detonation after the waves coalescenced was also less than that expected from the single shock Pop-plot data. The results of experimental shots 1281/1282 were also similar to the second set, however the detonation 
occurred approximately at the point of coalescence of the two waves. These results indicate that the often quoted 'rule of thumb', that build-up to detonation in doubly shocked explosive does not start until the waves coalesce [2], no longer applies in the situation where first shock generates significant hot-spot reaction.

Mader [14] reported that Dick imposed pre-shocks of 2.3 and $5.0 \mathrm{GPa}$ respectively on PBX-9502 explosive. In both cases, the detonation failed to propagate in the pre-shocked explosive. The pre-shock pressures were below the ignition threshold for PBX-9502. These results suggest that the desensitization occurred by mechanically compressing out the potential hot-spot sites, rather than by partial chemical reactions.

\section{Modelling Efforts}

Although some physics-based shock initiation models [15-17] have been reported to account for the desensitization phenomena, these models require huge computation resources. On the other hand, most of the phenomenological reactive flow models are incapable of simulating the desensitization induced by pre-shocking. Many attempts to include the desensitization effect in these models have been reported in the literature. The JTF model [18] accounts for this effect by restricting the creation of hot spots to the first shock only, and allowing a second shock to provide only adiabatic heating. The Wescott-Stewart-Davis (WSD) model [19] has been improved [20] to a temperature dependent form WSD (T). Although WSD (T) in the current form is incapable of predicting global desensitization, it is capable of predicting the reduction in the reactivity of the explosive with multi-shock compression. Mader used the multiple shock Forest-Fire model [21] to simulate the shock desensitization of Composition B resulting from the bow shock in metal jet penetration experiments [22]. Aminov et al. [23] developed a kinetic model, based on the activation energy of hot spots, to simulate the double-shock experiments [7] for LX-17 explosive. It was stated that the model described represented experiments not "worse" than the Lee-Tarver model. The CREST model [24] has been reported recently to predict desensitization phenomena [25], however, the model is still being improved upon at the Atomic Weapons Establishment (AWE), UK [26].

The standard ignition and growth Lee-Tarver model [27] is also incapable of predicting the desensitization effect by precursor shocks, since there is no mechanism to differentiate between a single and a double shock process. In this paper we focus on an adaptation of the Lee-Tarver ignition and growth model for simulation of desensitization in explosives. 


\subsection{Adapting the Lee-Tarver model}

The Lee-Tarver model employs separate temperature-dependent JWL equations of state (EOS) for both the unreacted explosive and the reaction products, and a reaction rate equation. The three term reaction rate equation for the standard model is of the form:

$$
\dot{\lambda}=R_{I}+R_{G_{1}}+R_{G_{2}},
$$

where

$$
\begin{aligned}
& R_{I}=I(1-\lambda)^{b}(\eta-1-a)^{x} \times H\left(\lambda_{\text {igmax }}-\lambda\right) \\
& R_{G_{1}}=G_{1}(1-\lambda)^{c} \lambda^{d} p^{y} \times H\left(\lambda_{G_{1} \max }-\lambda\right) \\
& R_{G_{2}}=G_{2}(1-\lambda)^{e} \lambda^{g} p^{z} \times H\left(\lambda-\lambda_{G_{2} \min }\right)
\end{aligned}
$$

and $H$ is a Heaviside function (equals one when the argument is positive, otherwise zero). The dot over $\lambda$ denotes its time derivative.

The standard Equation 3 does not account for dead pressing and thus results in the rapid reaction by a second shock. Several attempts have been made, all on ad hoc bases, to modify the Lee-Tarver ignition and growth model in order to reproduce the main features of some particular experiments.

Long ago, Tarver et al. [7] added a condition in the reaction rate equation to account for the desensitization of LX-17 by reflected shock. The condition was that if a critical range of pressures were applied to the explosive, the reaction rate was forced to zero. The calculated growth histories using the modified reaction rate equation agreed well for the reflected shock experiments, except for a few exceptions.

Whitworth et al. [28] described a simple model to predict the shock desensitization effect by modifying the reaction rate equation, making it dependent on the pre-shock pressure $P_{\text {shk }}$. In place of the local pressure $P$ in the reaction rate equation, $P=\min \left(P, P_{s h h}\right)$ was used to find the reaction rate. A qualitative agreement was reported for the calculations of Mulford's precursor shock experiment [5] using the modified reaction rate equation. Whitworth also applied his modified reaction rate equation for detonation quenching experiments. However, contrary to the experimental results [2], the rate of quenching increased with decreasing pressure of the precursor shock.

In order to model the qualitative features of the corner-turning failure in LX-17, Souers et al. [29] presented a failure model that cut off the reaction rate below certain detonation velocities. A single reaction rate equation was used, 
which was a stripped down version of the Lee-Tarver ignition and growth model, and the coefficients of this rate equation were different in different regions of the explosive. In a more recent approach, Souers et al. [30] later refined his model by employing a single reaction rate format, which varied for different pressure regimes in the explosive to simultaneously model dead zone, failure region and the detonation region in corner turning experiments. This model could reproduce various experimental features, including the detonation failure region in the corner turning experiments of LX-17.

DeOliveira et al. [31] proposed a modification in the Lee-Tarver ignition and growth model by introducing the desensitization rate $S$ as:

$$
S=M P(1-\varphi)(\varphi+\varepsilon)
$$

where $\varphi$ is the degree of desensitization, which varies from 0 to 1 . At $\varphi=0$, the material is in the normal or virgin state while $\varphi=1$ represents the completely desensitized explosive. $M$ is the rate constant and $\varepsilon$ is a small positive number to ensure some desensitization at $\varphi=0$ when $P>0$.

The critical compression in the ignition term of the original Lee-Tarver model is also prescribed as a function of $\varphi$. Thus the ignition term in reaction rate Equation 3 becomes:

$$
R_{I}=I(1-\lambda)^{b}(\eta-1-a(\varphi))^{x} \times H\left(\lambda-\lambda_{\text {igmax }}\right)
$$

where the modified critical compression $a(\varphi)$ is defined as:

$$
a(\varphi)=a_{0}(1-\varphi)+a_{1}(\varphi)
$$

where $1+a_{0}$ is the critical compression for ignition of the virgin material and $1+a_{1}$ is that for the completely desensitized material. The second change in the reaction rate equation proposed [31] was to change the switching limit of the growth term from 0 to $\lambda_{G_{1} \max }(\varphi)$, defined by $\lambda_{G_{1} \min }=\lambda_{c} \varphi$, where $\lambda_{c}>0$. Thus the modified growth term $R_{G_{1}, \varphi}$ for the desensitized explosive is defined as:

$$
R_{G_{1}, \varphi}=G_{1}(1-\lambda)^{c} \lambda^{d} p^{y} \times H\left(\lambda-\lambda_{G_{1} \min }(\varphi)\right) H\left(\lambda_{G_{1} \max }-\lambda\right)
$$

This modification requires four additional empirical constants to enable the ignition and growth model to account for the desensitization effect. Using the augmented model, DeOliveira et al. [31] qualitatively reproduced the main features of the corner turning experiments including the dead zones. It has been 
used by Tarver [32] for simulation of corner turning experiments, Jack-Rabbit experiments and detonation diffraction experiments [33].

The form factor $(1-\varphi) \varphi$ for the desensitization rate equation (Equation 4) seems similar to the form factor $(1-\lambda)^{c} \lambda^{d}$ of the growth terms in the original Lee-Tarver reaction rate equation, with $\lambda$ replaced by $\varphi$. However, we find no logic behind this similarity. The form factor $(1-\lambda)^{c} \lambda^{d}$ in the growth terms of the Lee-Tarver reaction rate equations not only forces the growth rate to zero at the start and end of the reaction but also corresponds to the inward and outward burning of the pores, and the exponents are set to match the calculation results with the results of the shock to detonation transition (SDT) experiments. While in the desensitization reaction rate equation, the form factor $(1-\varphi) \varphi$ is just meant to force the desensitization rate to zero at the start and at the end of the desensitization process. As the desensitization is assumed to be mainly caused by the collapsing of pores, and there is no reason to believe that the collapsing rate of the pores is lowest when the maximum number of pores is available at the start. Moreover, as shown in Figure 1, the form factor is maximum when the material is $50 \%$ desensitized, which implies that the desensitization rate is also maximum when the material is $50 \%$ desensitized.

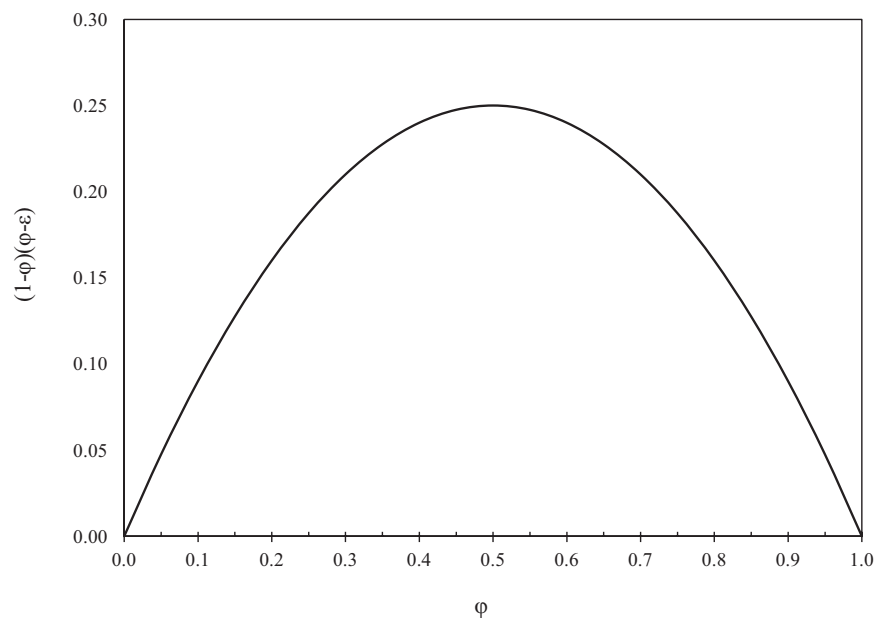

Figure 1. The form factor for the desensitization model proposed by DeOliveira [31].

Another issue with the desensitization rate is its linear dependence on the pre-shock pressure. Using their experimental data, Campbell and Travis [2] proposed a relation indicating that PBX9404 would be fully desensitized for $P^{2.2} \tau=1150\left(\mathrm{kbar}^{2.2} \cdot \mu \mathrm{s}\right)$, where $P$ is the pre-shock pressure and $\tau$ is the 
time required for desensitization. This implies that the desensitization rate is proportional to the 2.2 power of $P$, and not linearly proportional to $P$.

The method of finding the parameters of the DeOliveira's model is also described in reference [31]. DeOliveira guessed the values of the parameters $\varepsilon$ and $M$. The parameter $\varepsilon$ was assumed to be 0.0001 to yield a non-zero desensitization rate at the start of the desensitization process when $(\varphi=0)$. In addition, $M$ was guessed to be 1000 to simulate the corner turning experiments. Later on Tarver [32] used $M=1000$ to simulate the Jack-Rabbit experiments and the detonation diffraction experiments. However, Tarver [32] observed that the desensitization rate was actually higher than predicted by the calculations using the above desensitization model with $M=1000$. However in gas gun experiments by Vandersall et al. [11], it was found that $M$ should be increased from 1000 to 1200 , eventually increasing the desensitization rate, to reproduce the experimental pressure history at various gauge points.

\subsection{The present approach}

In continuation of the effort in adapting the ignition and growth model for the shock desensitization problem, we followed DeOliveira's approach with a few changes. Our model assumes that the shock desensitization takes place when the pre-shock pressure is in a specific range for the explosive. For example, Wescott et al. [34] proposed that desensitization of PBX-9502 explosive takes place between around 1 and $6 \mathrm{GPa}$. We also assume that shock desensitization is a time dependent process, which is an experimentally established fact as discussed in the previous section. We also utilize the $P^{m} \tau$ criterion $(m=2.2$ for PBX-9404 explosive) suggested by Campbell and Travis [2] to measure the degree of desensitization.

Firstly, we define the degree of desensitization $\varphi$ as:

$$
\varphi=\frac{1}{P^{m} \tau} \int_{t_{1}}^{t} P^{m}(t) d t
$$

where the time $t_{l}$ corresponds to the pressure $P_{l}$ (the Hugoniot elastic limit, below which it is assumed that no damage is done to the explosive and after which the desensitization begins) and the upper limit of the above integral is time $t$, corresponding to the pressure $P_{2}$ (the threshold pressure for ignition). At $P=P_{1}, \varphi=0$ and at $P=P_{2}, \varphi=1$.

The degree of desensitization increases with pressure, as in Equation 8, and the desensitization rate also increases (Figure 2). However, it is suggested that 
although the desensitization increases with pressure, the desensitization rate decreases with increase in pressure in the specific interval $\left(P_{1}, P_{2}\right)$. Therefore, we modified slightly the degree of desensitization determined by Equation 8 as follows:

$$
\phi=\sin \left(\frac{\pi}{2} \varphi^{n}\right)
$$

Exponent $n$ can be used to adjust the rate of desensitization with pressure. The modified degree of desensitization $\phi$ (with $n=0.5$ ) was used in our calculations. $\phi$ also varies, in a similar manner to $\varphi$, i.e. at $P=P_{1}, \phi=0$ and at $P=P_{2}, \phi=1$. However the rate of desensitization decreases with increase in pressure (Figure 2).

Secondly, the critical compression is increased to account for the loss of the potential hot spot generation sites, and is defined as:

$$
a(\varphi)=a+\phi C_{l},
$$

where $a$ is the critical compression of the virgin and the pre-shocked material and $C_{I}$ is an empirical constant adjusted to inhibit the ignition term in the reaction rate equation (hence inhibiting the detonation in the pre-shocked explosive).

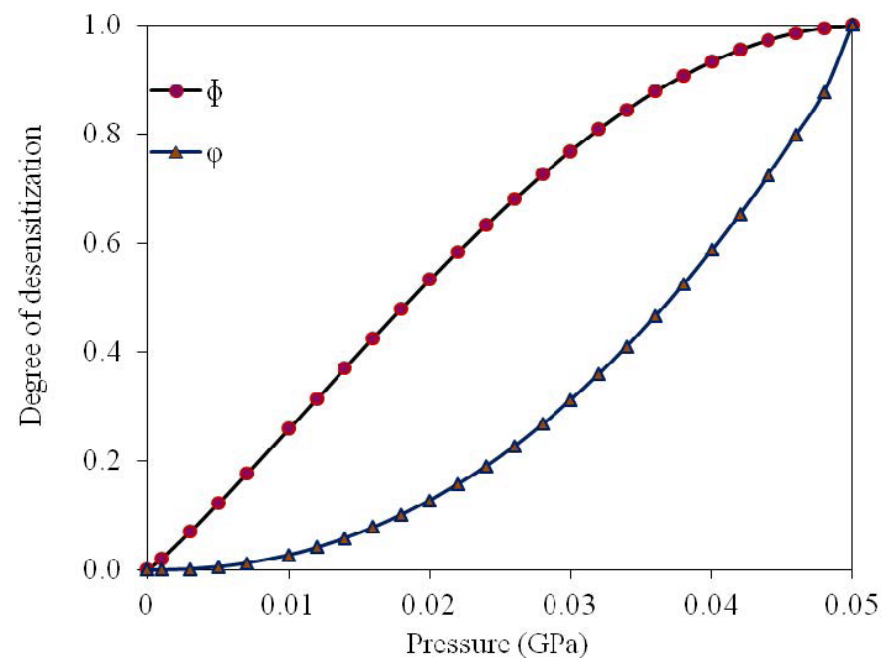

Figure 2. The degree of desensitization as a function of shock pressure calculated using two kinds of functions as given in Equations 8 and 9. 
The growth term coefficients were also adjusted to control the growth of the reaction in the desensitized or partially desensitized explosive, as follows:

$$
\begin{aligned}
& G_{1}(\varphi)=(1-\phi) G_{1}, \\
& G_{2}(\varphi)=(1-\phi) G_{2}
\end{aligned}
$$

It should be noticed that if the explosive is partially desensitized $(\phi<1)$ by the first shock, it would be possible to have some reaction on the passage of the higher-pressure (second) shock. This feature helped us to reproduce the experimental results of the EDC37 double shock experiments [12,13], as will be described in the next section.

The parameter $C_{I}$ is the control parameter for the augmented/adapted model; its value for a typical explosive can be estimated by empirically fitting the experimental data. In our calculations involving PBX-9404, we estimated $C_{I}$ to be 0.1 .

\section{Modelling and Simulation}

The proposed extension was implemented in LS-DYNA to study the shock desensitization experiments.

\subsection{Implementation in LS-DYNA}

The Lee-Tarver ignition and growth model is one of the popular reactive burn models in use today and has been embedded already in various commercial hydrocodes, such as LS-DYNA, ANSYS AUTODYN, Abaqus and many others. By utilising the user equation of state option [35], we have implemented the ignition and growth model in LS-DYNA for the present study. First of all we have validated the newly implemented Lee-Tarver model by comparing the simulation results for simple problems using both the inbuilt and the newly implemented Lee-Tarver model. After validation, we modified the model as described in the previous section to simulate the double shock experiments. The degree of desensitization was stored as a history variable for every computational element and was updated after every time interval. During the execution of the subroutine, it is determined initially whether the input pressure $(P)$ lies in the range for desensitization $\left(P_{1}<P<P_{2}\right)$. If so, then the degree of desensitization is determined using Equations 8 and 9, and stored in the history. When the second wave arrives, the modified parameters determined by Equations 10 and 11 are used to calculate the reaction rate using Equation 3. 
Following implementation, this model can now be used as an equation of state for any explosive, to simulate a variety of situations involving explosives in the simulation environment of LS-DYNA. The new parameters $P_{1}, P_{2}, m, P^{m} \tau$ and $C_{I}$ need to be defined along with other Lee-Tarver equation of state parameters in the input file. By setting the value of $C_{I}$ to zero, the newly modified Lee-Tarver model functions as the traditional Lee-Tarver Model.

\subsection{Simulation of double shock experiments}

The schematic diagram for the simulation setup is given in Figure 3. A threedimensional mesh with constraints applied on the lateral dimensions (along the $\mathrm{x}$ - and y-axes) was used. The mesh resolution was 20 elements per $\mathrm{mm}$. The length of the explosive was taken as $20 \mathrm{~mm}$. The pressure history was measured at various gauge points inside the explosive; the gauges were $0.5 \mathrm{~mm}$ apart. The series of double shock initiation experiments $[12,13]$ on an HMX based explosive EDC37 was chosen for the simulation and validation of the newly implemented model, because these experiments reveal a broader range of features involved in the shock desensitization process of an explosive. Figures 10 and 11 in reference [13] are experimental particle velocity histories at different gauge positions inside the EDC37 explosive for shots 1175/76 and shots 1191/92 respectively. For simulation of these reported experiments [13], two pressure pulses, where the second pressure pulse was delayed by the experimental time for each shot, were applied on one side of the explosive as initial conditions, as shown in Figure 3. Since the $P^{m} \tau$ criterion for EDC37 was not available, we used another HMX-based explosive (PBX-9404) instead. The use of PBX-9404 in place of EDC37 in the simulation is not expected to harm the qualitative nature of the results, since our purpose here is just to verify the availability of the model for simulating double shock experiments. Moreover, the HMX contents in the two explosives are similar (94\% and 91\% in PBX-9404 and EDC37, respectively). For the same density of the two explosives, some authors [36, 37] have used the same reaction products JWL equation of state for both explosives. The detonation velocities of the two explosives are also similar. The parameter set for the Lee-Tarver model of PBX-9404 with a two-term reaction rate equation was taken from LLNL report [38] and is reproduced in Table 1. 
Table 1. Physical properties and parameters for the augmented Lee-Tarver model

\begin{tabular}{|c|c|c|c|}
\hline \multirow{5}{*}{\multicolumn{2}{|c|}{ Physical properties }} & Density $\left[\mathrm{g} / \mathrm{cm}^{3}\right]$ & 1.842 \\
\hline & & $C_{v}$ products $[\mathrm{Mbar} / \mathrm{K}]$ & $1.0 \mathrm{E}-5$ \\
\hline & & $C_{v}$ reactants $[\mathrm{Mbar} / \mathrm{K}]$ & $2.7813 \mathrm{E}-5$ \\
\hline & & Shear Modulus [Mbar] & 0.045 \\
\hline & & Temperature [K] & 298 \\
\hline \multirow{5}{*}{\multicolumn{2}{|c|}{$\begin{array}{l}\text { Reactants } \\
\text { JWL } \\
\text { EOS parameters }\end{array}$}} & $A[\mathrm{Mbar}]$ & 9522 \\
\hline & & $B$ [Mbar] & -0.05944 \\
\hline & & $R_{1}$ & 14.1 \\
\hline & & $R_{2}$ & 1.41 \\
\hline & & $\Omega$ & 0.3 \\
\hline \multirow{5}{*}{\multicolumn{2}{|c|}{$\begin{array}{l}\text { Product } \\
\text { JWL } \\
\text { EOS parameters }\end{array}$}} & $A[\mathrm{Mbar}]$ & 8.524 \\
\hline & & $B$ [Mbar $]$ & 0.1802 \\
\hline & & $R_{1}$ & 4.6 \\
\hline & & $R_{2}$ & 1.3 \\
\hline & & $\Omega$ & 0.38 \\
\hline \multirow{15}{*}{ Reaction rates } & \multirow{4}{*}{ Ignition term } & parameter $I\left[\mu \mathrm{s}^{-1}\right]$ & 44 \\
\hline & & reaction ratio exp. & 0.222 \\
\hline & & critical compression. $a$ & 0.01 \\
\hline & & compression exp. $x$ & 4.0 \\
\hline & \multirow{4}{*}{ First growth term } & $G_{1}\left[\mathrm{Mbar}^{-\mathrm{y}} \mu \mathrm{s}^{-1}\right]$ & 0 \\
\hline & & reaction ratio exp. $c$ & 0 \\
\hline & & reaction ratio exp. $d$ & 0 \\
\hline & & pressure exp. $y$ & 0 \\
\hline & \multirow{4}{*}{ Second growth term } & $G_{2}\left[\mathrm{Mbar}^{-\mathrm{z}} \mu \mathrm{s}^{-1}\right]$ & 495 \\
\hline & & reaction ratio exp. $e$ & 0.222 \\
\hline & & reaction ratio exp. $g$ & 0.667 \\
\hline & & pressure exp. $z$ & 2.0 \\
\hline & \multirow{3}{*}{ Limits } & $\lambda_{\text {igmax }}$ & 0.3 \\
\hline & & $\lambda_{\text {GImax }}$ & 1 \\
\hline & & $\lambda_{G 2 \min }$ & 0 \\
\hline \multirow{5}{*}{\multicolumn{2}{|c|}{ Augmented model parameters }} & $P_{1}[\mathrm{Mbar}]$ & 0.01 \\
\hline & & $P_{2}[\mathrm{Mbar}]$ & 0.05 \\
\hline & & $M$ & 2.2 \\
\hline & & $P^{m} \tau\left[\mathrm{Mbar}^{2.2} \cdot \mu \mathrm{s}\right][2]$ & $2.864 \mathrm{E}-4$ \\
\hline & & $C_{I}$ & 0.10 \\
\hline
\end{tabular}




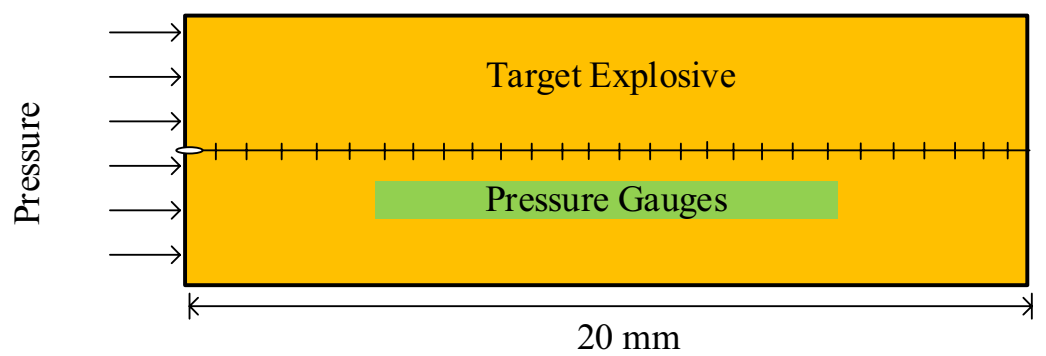

Figure 3. Simulation setup for double shock studies.

The simulation results are plotted in Figures 4 and 5 and in Figures 6 and 7 for the original and modified Lee-Tarver models (for shots 1175 and 1191 $[12,13]$ respectively). The comparison between the presently calculated and the experimental run distance to detonation for the three experimental shots is given in Table 2 below.

Table 2. The calculated and experimental results of double shock experimental details for shots 1175,1194 and 1281

\begin{tabular}{|c|c|c|c|c|c|c|c|c|}
\hline \multirow[b]{3}{*}{ Shot } & \multirow{3}{*}{$\begin{array}{c}\text { First } \\
\text { shock } \\
\text { pressure } \\
{[\mathrm{GPa}]}\end{array}$} & \multirow{3}{*}{$\begin{array}{c}\text { Time } \\
\text { between } \\
\text { shocks } \\
{[\mu \mathrm{s}]}\end{array}$} & \multirow{3}{*}{$\begin{array}{c}\text { Second } \\
\text { shock } \\
\text { pressure } \\
{[\mathrm{GPa}]}\end{array}$} & \multirow{3}{*}{$\begin{array}{c}\text { Coalescence } \\
\text { distance } \\
{[\mathrm{mm}]}\end{array}$} & \multicolumn{4}{|c|}{$\begin{array}{l}\text { Detonation run distance } \\
{[\mathrm{mm}]}\end{array}$} \\
\hline & & & & & \multicolumn{2}{|c|}{$\begin{array}{c}\text { From } \\
\text { front face }\end{array}$} & \multicolumn{2}{|c|}{$\begin{array}{c}\text { From } \\
\text { coalescence }\end{array}$} \\
\hline & & & & & $\frac{\overrightarrow{0}}{\frac{\vec{\pi}}{\Xi}}$ & 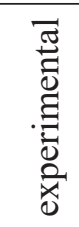 & $\begin{array}{l}\frac{\overrightarrow{0}}{\tilde{J}} \\
\frac{\vec{J}}{\tilde{0}} \\
\frac{\tilde{J}}{0}\end{array}$ & 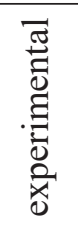 \\
\hline 1175 & 2.94 & 0.550 & 6.2 & 5.0 & 11.96 & 11.5 & 6.18 & 6.5 \\
\hline 1194 & 3.93 & 0.676 & 8.54 & 4.5 & 9.43 & 8.0 & 2.43 & 3.5 \\
\hline 1281 & 4.15 & 1.014 & 9.06 & 9.5 & 10 & 10 & $<1$ & 0.5 \\
\hline
\end{tabular}




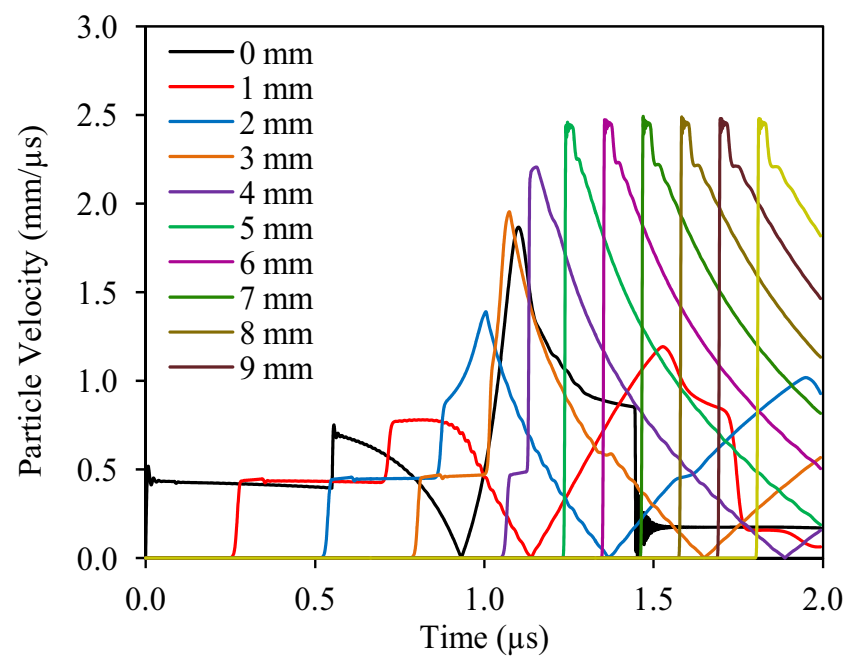

Figure 4. Particle velocity history plots calculated by the Lee-Tarver model for double shock experiments at different gauge positions inside EDC37 explosive for shots 1175/1176.

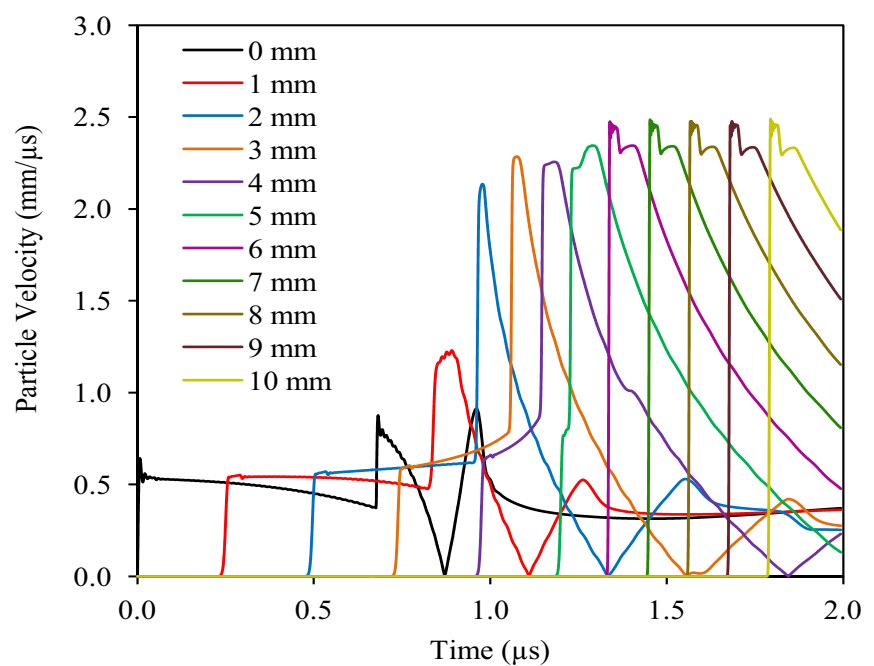

Figure 5. Particle velocity history plots calculated by the Lee-Tarver model for double shock experiments at different gauge positions inside the EDC37 explosive for shots 1194/1195. 


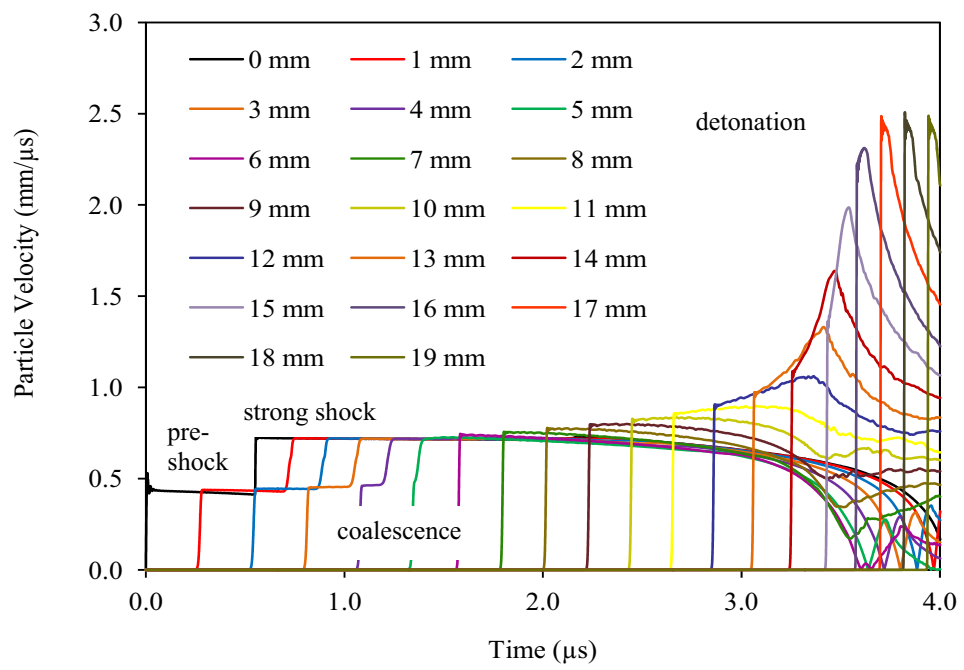

Figure 6. Particle velocity history plots calculated by the augmented LeeTarver model for double shock experiments at different gauge positions inside the EDC37 explosive for shots 1175/1176.

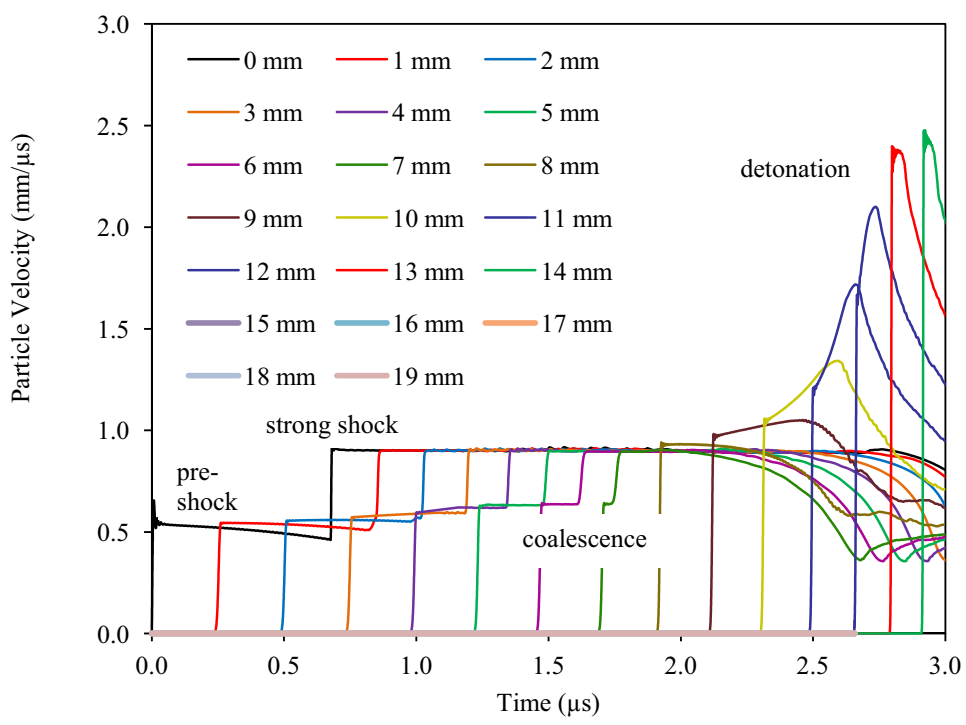

Figure 7. Particle velocity history plots calculated by the augmented LeeTarver model for double shock experiments at different gauge positions inside the EDC37 explosive for shots 1194/1195. 


\subsection{Reflected shock experiments}

Tarver et al. [7] performed reflected shock experiments to study the response of LX-17 explosive. However for our calculations of reflected shock experiments, we used PBX-9404, again because the desensitization criterion for LX-17 was not available. Our aim here was to verify whether the augmented Lee-Tarver model is able to qualitatively reproduce the main features of the reflected shock experiments.

We modelled the problem by using a one-dimensional mesh of $20 \mathrm{~mm}$ length (Figure 8). The meshing details are the same as in Section 4.2. A sustained pressure pulse $(3.93 \mathrm{GPa})$ was applied at one side of the explosive, being insufficient to cause detonation in the $20 \mathrm{~mm}$ length of the explosive. At the other edge of the mesh a perfectly reflecting boundary was applied. A high pressure $(15.45 \mathrm{GPa})$ was caused by the reflection of the shock from the perfectly reflecting boundary.

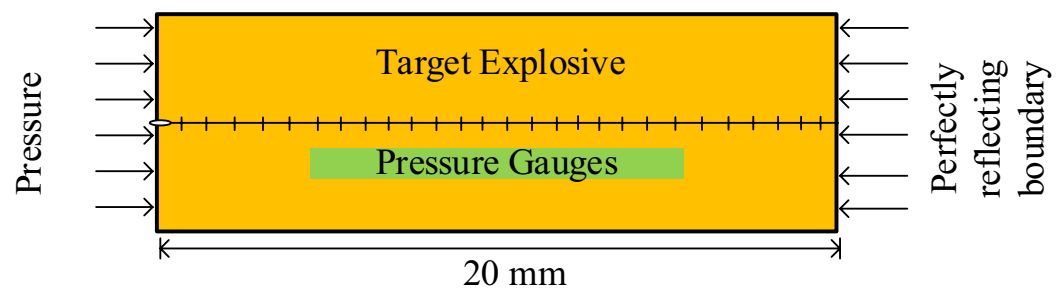

Figure 8. Simulation setup for reflected shock studies.

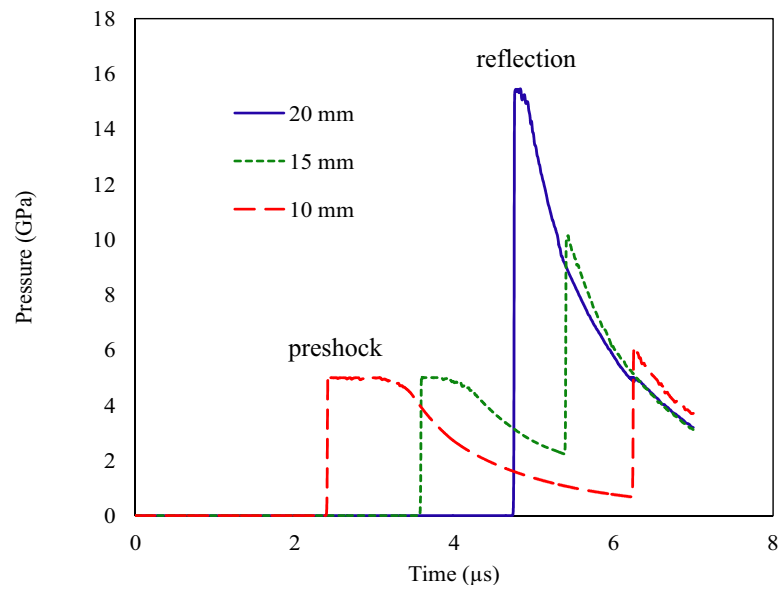

Figure 9. Pressure history at 3 gauge points inside the explosive at 10.0, 15.0 and $20.0 \mathrm{~mm}$, respectively. 
The pressure history at 3 gauge points inside the explosive, at 10.0, 15.0 and $20 \mathrm{~mm}$ respectively (the $20 \mathrm{~mm}$ gauge was at the highest pressure peak, while the $10.0 \mathrm{~mm}$ gauge was at the lowest peak) is shown in Figure 9.

\subsection{Detonation quenching}

Although the detonation quenching experiments were performed with LX-17, we used PBX-9404, again because the desensitization criterion for LX-17 was not available. We modelled the problem by using a one-dimensional mesh of $20 \mathrm{~mm}$ length (Figure 10). The meshing details are as described earlier. A sustained pressure pulse $(3.0 \mathrm{GPa})$ was applied at one side of the explosive. This is insufficient to cause detonation in the $20 \mathrm{~mm}$ of the explosive. After a delay of $2.5 \mu \mathrm{s}, 9.0 \mathrm{GPa}$ pressure was applied as another boundary condition at the other edge of the mesh.

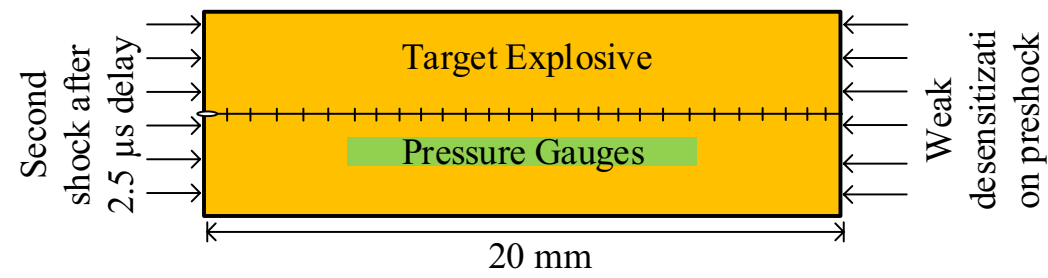

Figure 10. Simulation setup for detonation quenching studies.

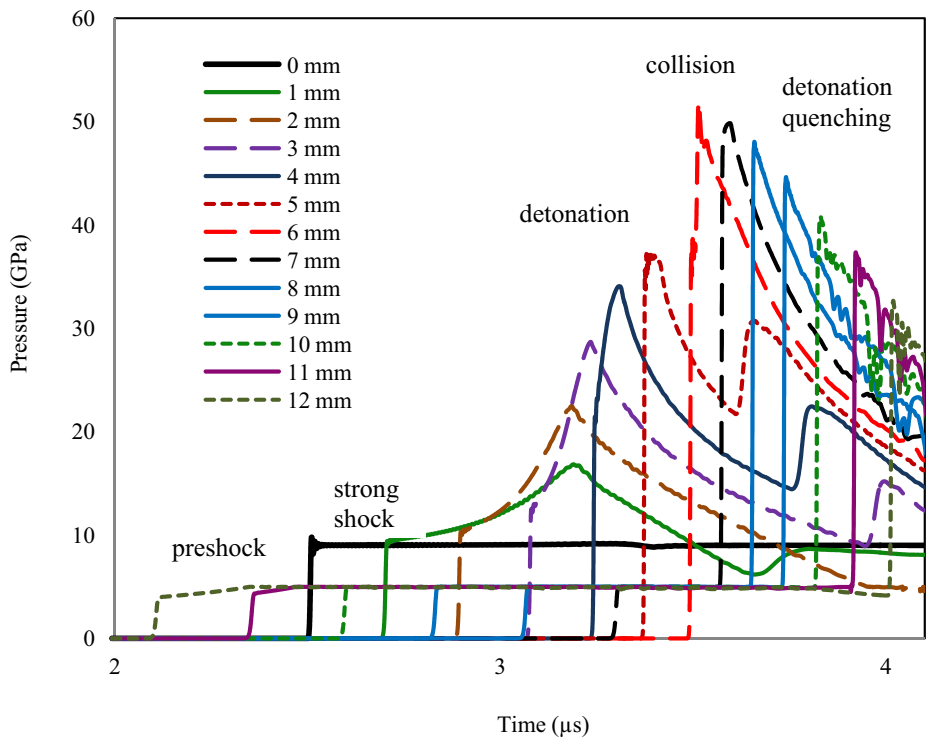

Figure 11. Pressure history at different gauge points inside the explosive. 
The pressure history at various gauge points inside the explosive is shown in Figure 11, where the collision of the weak shock with the detonation wave is observed at the $6 \mathrm{~mm}$ gauge, after which the detonation wave was quenched, as can be observed by the continuously decreasing magnitude of the pressure peaks at the later gauge points.

\section{Results and Discussion}

As can be noticed from the experimental particle velocity plots for the shots 1175/76 given in Figure 10 in reference [13], no reaction is observed in the first wave, and the run distance to detonation after wave coalescence is as expected from the single shock Pop-plot for EDC37 (Figure 34 in [13]). However in the second set of experiments (shots \#1191/92, plotted in Figure 11 in [13]), where the explosive was subjected to a $3.92 \mathrm{GPa}$ shock followed $0.6 \mu$ s later by a main shock of $8.56 \mathrm{GPa}$ strength, significant reaction occurred in the first wave and even more in the second wave. The run distance to detonation after wave coalescence was also smaller than that expected from the single shock Pop-plot data. The third set of experiments (shots 12881/2, plotted in Figure 12 in [13]) is similar to shots \#1194/95 with slightly higher pressure and a greater time interval between the two shocks, the detonation occurred close to wave coalescence. The general principle, as formulated by Davis [39], states that the run distance to detonation $(L)$ is defined as $L=x_{0}+r_{d d}$, where $x_{0}$ is the distance at which the waves coalesce and $r_{d d}$ is the run distance to detonation for a single shock in the virgin explosive. The results indicate that this rule no longer applies in the situation where the first shock generates significant hot-spot reaction. The simulation results shown in Figures 6 and 7 are in accordance with this conclusion. The Lee-Tarver model has been modified to account for the desensitization effects. The results of the experiment [13] have been reproduced by using the modified Lee-Tarver model for the first time, at least qualitatively. This is an improvement on DeOliveira's approach used to simulate the same experiment [40]. The calculated run distance to detonation is also comparable with the experimental results. The original Lee-Tarver model is not capable of reproducing the desensitization effects in an explosive, as can be seen in Figure 4 and in Figure 5, which are the plots for the simulation of the experimental shots 1175 and 1194 respectively using the original Lee-Tarver model. The results obtained after modification of the model (plotted in Figures 6 and 7 for the two shots, respectively) are qualitatively in good agreement with the experimental curves (Figures 10 and 11 in [13]).

The new model was also used to simulate reflected shock experiments. The 
results (Figure 9) are in accordance with the trend observed in experiments that the reflected high pressure shock (14.5 GPa in this case) fails to detonate the explosive because it was desensitized by the earlier $3.93 \mathrm{GPa}$ shock. The pressure histories at various gauge points show that the pressure keeps on decreasing with the passage of the reflected shock, and the highest pressure is noted close to the reflecting surface. It should be noted that the pressure at the reflecting boundary (at the $20 \mathrm{~mm}$ gauge) is not merely double the incident pressure, as one might guess from a non-reacting shock travelling in the explosive being reflected from a perfectly reflecting boundary. The weak shock near the reflecting boundary did not have sufficient time to completely desensitize the explosive. Since the desensitization model used here is based on the desensitization criterion, which is a function of both pressure and time, the degree of desensitization is a minimum near the reflecting boundary, and keeps on increasing with distance from the reflecting boundary. Therefore, some reaction occurs at the boundary, eventually increasing the pressure. However, the pressure drops as the shock travels away from the boundary because the degree of desensitization increases, resulting in a decrease in the reaction. This in accordance with the experimental results presented by Tarver et al. [7]. As discussed above, Tarver used a $20 \mathrm{~mm}$ thick LX-17 disc that was impacted by different types of flyer. The pressure histories were measure at 5,10 and $15 \mathrm{~mm}$ depths. The pressure measured at the $15 \mathrm{~mm}$ depth was approximately double the incident pre-shock pressure. Obviously, the pressure at the reflecting surface at $20 \mathrm{~mm}$ depth would have been higher than at $15 \mathrm{~mm}$ depth. As shown in Figure 11, the present model also yields approximately double the pressure at the $15 \mathrm{~mm}$ depth.

Another test for the new model is the simulation of detonation quenching in shock colliding experiments. It is obvious from the results shown in Figure 11 that the fully developed detonation is extinguished when it enters the precompressed (dead) explosive which was earlier desensitized by a weak (3.93 GPa) shock. The pressure histories at various gauge points prove that the pressure continuously decreases in the direction of the detonation wave propagation after the shocks collide with each other. The highest pressure is at the point of collision of the waves.

\section{Conclusions}

The present modification of the Lee-Tarver model can be used to account for the desensitization effects. The results of the experiment [13] have been reproduced, at least qualitatively, by using the modified Lee-Tarver model. The run distance 
to detonation is also comparable to the experimental results.

The model proposed here is also based on the degree of desensitization, which is calculated on the basis of the $P^{m} \tau$ criterion which has been determined for some explosives by double shock initiation experiments [2]. After implementation in LS DYNA, this model is available to study a variety of problems involving explosives, especially desensitization by pre-shocking. Our model qualitatively reproduces the experimental results of EDC37 double shock experiments [12, 13]. The simulation results presented here successfully reproduce the main features of the desensitization of heterogeneous explosives by double shock, reflected shock and detonation quenching processes respectively, and provide qualitative agreement with the experimental results. The extension does not change the functionality of the original Lee-Tarver model, unless the explosive is subjected to a weak pre-shock, which desensitizes the explosive. Nor does it change the ignition and growth parameters of an explosive, except that a few more empirical constants have to be added to simulate the desensitization of the explosive.

The desensitization criterion for other heterogeneous explosives needs to be determined for further verification and calibration of the model for those explosives. Further desensitization experiments will be of extreme value for the validation/improvement of the model.

\section{Acknowledgements}

The authors are grateful to Prof. Zhang Zhenyu of the College of Science, National University of Defense Technology, Changsha, P. R. China, for helping us in the implementation of the user equation of state subroutine in LS-DYNA. This paper was supported by the National Natural Science Foundation of China (Grant No. 11272059, 11221202 and 11390362), Program for New Century Excellent Talents in University (Grant No. NCET-12-0037), and the project of State Key Laboratory of Explosion Science and Technology (Beijing Institute of Technology) under Grant No. YBKT106. Author \#1 is supported by the Higher Education Commission of Pakistan under OSS Scheme, Phase,2, Batch,4.

\section{References}

[1] Campbell A.W., Davis W.C., Ramsay J.B., Travis J.R., Shock Initiation of Solid Explosives, Phys. Fluids (1958-1988), 1961, 4(4), 511-521.

[2] Campbell A.W., Travis J.R., The Shock Desensitization of PBX-9404 and Composition B-3, Proc., Symp. Detonation, $8^{\text {th }}$, Albuquerque, New Mexico, July 15-19, 1985. 
[3] Chick M.C., Hatt D.J., The Initiation of Covered Composition B by a Metal Jet, Propellants Explos. Pyrotech., 1983, 8(4), 121-126.

[4] Setchell R.E., Effects of Precursor Waves in Shock Initiation of Granular Explosives, Combust. Flame, 1983, 54(1-3), 171-182.

[5] Mulford R.N., Sheffield S.A., Alcon R.R., Preshock Desensitization of PBX Explosives, AIP Conf. Proc., 1994, 309(1), 1405-1408.

[6] Mulford R.N., Swift D.C., Reactive Flow Models for the Desensitization of High Explosive, AIP Conf. Proc., 2000, 505(1), 895-898.

[7] Tarver C.M., Cook T.M., Urtiew P.A., Tao W.C., Multiple Shock Initiation of LX-17, Proc., Symp. Detonation, 10 $0^{\text {th }}$, Boston, Massachusetts, July 12-16, 1993.

[8] Tarver C.M., Hallquist J.O., Erickson L.M., Modelling Short Pulse Duration Shock Initiation of Solid Explosives, Proc., Symp. Detonation, $8^{\text {th }}$, Albuquerque, NM, 1985.

[9] Bordzilovskii S.A., Karakhanov S.M., Desensitization of Pressed RDX/Paraffin and HMX/Paraffin Compounds by Multiple Shock Waves, Combust., Explos. Shock Waves (Engl. Transl.), 1995, 31(2), 227-235.

[10] Bat'kov Y.V., Glushak B.L., Novikov S.A., Desensitization of Pressed Explosive Compositions Based on TNT, RDX, and HMX under Double Shock-wave Loading, Combust., Explos. Shock Waves (Engl. Transl.), 1995, 31(4), 482-485.

[11] Vandersall K.S., Garcia F., Tarver C.M., Fried L.E., Shock Desensitization Experiments and Reactive Flow Modeling on Self-sustaining LX -17 Detonation Waves, Proc., Symp. Detonation, 14 $4^{\text {th }}$, San Francisco, CA, July 13-18, 2014.

[12] Gustavsen R.L., Sheffield S.A., Alcon R.R., Winter R.E., Taylor P., Salisbury D.A., Double Shock Initiation of the HMX Based Explosive EDC-37, AIP Conf. Proc., 2002, 620(1), 999-1002.

[13] Winter R.E., Sorber S.S., Salisbury D.A., Taylor P., Gustavsen R., Sheffield S., Alcon R., Experimental Study of the Shock Response of an HMX-based Explosive, Shock Waves, 2006, 15(2), 89-101.

[14] Mader C.L., LASL PHERMEX Data, Vol. III, University of California Press, Berkeley, California, 1980.

[15] Plotard J.P., Belmas R., Nicollet M., Leroy M., Effect of a Preshock on the Initiation of HMX, TATB and HMX/TATB Compositions, Proc., Symp. Detonation, $10^{\text {th }}$, July 12-16, 1993.

[16] Hussain T., Liu Y., Huang F.-L., Duan Z.-P., Modeling and Simulation of Preshock Desensitization in Heterogeneous Explosives using a Mesoscopic Reaction Rate Model, Simulation, 2015, 91(11), 980-988.

[17] Duan Z.-P., Wen L.-J., Liu Y., Ou Z.-C., Huang F.-L., Zhang Z.-Y., A Pore Collapse Model for Hot-spot Ignition in Shocked Multi-component Explosives, Int. J. Nonlin. Sc. Num. Sim., 2010, 11 (Supplement), 19-23.

[18] Johnson J.N., Tang P.K., Forest C.A., Shock Wave Initiation of Heterogeneous Reactive Solids, J. Appl. Phys., 1985, 57(9), 4323-4334.

[19] Wescott B.L., Stewart D.S., Davis W.C., Equation of State and Reaction Rate for Condensed-phase Explosives, J. Appl. Phys., 2005, 98(5), 053514. 
[20] Desbiens N., Matignon C., Sorin R., Temperature-based Model for Condensedphase Explosive Detonation, J. Phys.: Conf. Ser., 2014, 500(15), 152004.

[21] Mader C.L., Numerical Modeling of Explosives and Propellants, $3^{\text {rd }}$ ed., CRC Press, Taylor \& Francis, 2008; ISBN 9781420052381.

[22] Mader C.L., Modeling Shock Desensitization of Composition B Explosive, J. Energ. Mater., 2014, 32, 80-87.

[23] Aminov Y.A., Es'kov N.S., Nikitenko Y.R., Modeling of Double Shock Initiation of TATB-base Explosives, Proc., Symp. Detonation, $12^{\text {th }}$, San Diego, California, 2002.

[24] Handeley C.A., CREST Reactive Flow Model, Proc., Symp. Detonation, $13^{\text {th }}$, Norfolk, VG, July 23-28, 2006.

[25] Whitworth N.J., CREST Modelling of PBX 9502 Corner Turning Experiments at Different Initial Temperatures, J. Phys.: Conf. Ser., 2014, 500(5), 052050.

[26] Lambourn B.D., James H.R., The Relation between Reaction Rate and Shock Strength - A Possible Second Order Improvement to the CREST Reactive Burn Model, AIP Conf. Proc., 2012, 1426(1), 591-594.

[27] Lee E.L., Tarver C.M., Phenomenological Model of Shock Initiation in Heterogeneous Explosives, Phys. Fluids (1958-1988), 1980, 23(12), 2362-2372.

[28] Whitworth N.J., Maw J.R., Modeling Shock Desensitisation of Heterogeneous Explosives, AIP Conf. Proc., 1996, 370(1), 425-428.

[29] Souers P.C., Andreski H.G., Cook C.F., Garza R., Pastrone R., Phillips D., Roeske F., Vitello P., Molitoris J.D., LX-17 Corner-turning, Propellants Explos. Pyrotech., 2004, 29(6), 359-367.

[30] Souers P.C., Andreski H.G., Batteux J., Bratton B., Cabacungan C., Cook C.F., Fletcher S., Garza R., Grimsley D., Handly J., Hernandez A., McMaster P., Molitoris J.D., Palmer R., Prindiville J., Rodriguez J., Schneberk D., Wong B., Vitello P., Dead Zones in LX-17 and PBX 9502, Propellants Explos. Pyrotech., 2006, 31(2), 89-97.

[31] DeOliveira G., Kapila A., Schwendeman D., Bdzil J., Henshaw W., Tarver C., Detonation Diffraction, Dead Zones and the Ignition-and-growth Model, Proc., Symp. Detonation, $13^{\text {th }}$, Norfolk, Virginia, July 23-28, 2006.

[32] Tarver C.M., Corner Turning and Shock Desensitization Experiments Plus Numerical Modeling of Detonation Waves in the Triaminotrinitrobenzene Based Explosive LX-17, J. Physical Chem. A, 2010, 114(8), 2727-2736.

[33] Tarver C.M., Modeling Detonation Experiments on Triaminotrinitrobenzene (TATB)-based Explosives LX-17, PBX 9502, and Ultrafine TATB, J. Energ. Mater., 2012, 30(3), 220-251.

[34] Wescott B.L., Stewart D.S., Davis W.C., Modeling Detonation Differaction and Dead Zones in PBX-9502, Proc., Symp. Detonation, $13^{\text {th }}$, Norfolk, Virginia, July 23-28, 2006.

[35] Hallquist J.O., LS-DYNA Keyword User's Manual, v. 971, Livermore, CA, Livermore Software Technology Corporation, 2007; ISBN 0-9778540-2-7.

[36] Whitworth N., Mathematical and Numerical Modelling of Shock Initiation in 
Heterogeneous Solid Explosives, PhD Thesis, Cranfield University, 2008.

[37] Belmas R., Plotard J.P., Bianchi C., A Physical Model of Shock to Detonation Transition in Heterogeneous Explosives, Proc., Symp. Detonation, $10^{\text {th }}$, July 12-16, 1993.

[38] Souers P.C., Haselman L.C.J., Detonation Equation of State at LLNL, 1993, UCRLID-116113, Livermore, Lawrence Livermore National Laboratories, 1994.

[39] Davis W.C., Shock Desensitizing of Solid Explosive, Proc., Symp. Detonation, $14^{\text {th }}$, Coeur d'Alene, Idaho, April 11-16, 2010.

[40] DeOliveira G., Numerical Studies of the Behaviour of Heterogeneous Explosives Using the Ignition-and-growth Model, PhD Thesis, New York, Rensselaer Polytechnic Institute, 2006. 
